¿Todo lo sólido se desvanece en cuarentena?

Garriga, María Cristina; Pappier, Viviana; Cuesta, Virgina; Linare, Cecilia; Rocha, Milagros

ISSN 2469-0090 | https://doi.org/10.24215/24690090e026

https://revistas.unlp.edu.ar/TrayectoriasUniversitarias

Universidad Nacional de La Plata

La Plata | Buenos Aires | Argentina

\title{
¿Todo lo sólido se desvanece en cuarentena?
}

\section{Does all solid fade in quarantine?}

\author{
Garriga, María Cristina \\ https://orcid.org/0000-0002-2880-0734 \\ pinagarriga@yahoo.com.ar \\ Universidad Nacional de La Plata | Argentina \\ Cuesta, Virgina \\ https://orcid.org/0000-0001-6056-7891 \\ virginia.cuesta@gmail.com \\ Universidad Nacional de La Plata | Argentina
}

\author{
Pappier, Viviana \\ https://orcid.org/0000-0002-7530-6296 \\ vpappier@yahoo.com \\ Universidad Nacional de La Plata | Argentina \\ Linare, Cecilia \\ https://orcid.org/0000-0003-3290-5413 \\ cecilialinare@gmail.com \\ Universidad Nacional de La Plata | Argentina
}

\author{
Rocha, Milagros \\ https://orcid.org/0000-0002-5361-0211 \\ milagrosmrocha@gmail.com \\ Universidad Nacional de La Plata | Argentina
}

\section{RESUMEN}

Las certezas, o por lo menos algunas desde donde partimos para pensar el espacio del aula, se encuentran en modo "recalculando". La incertidumbre nos invade, nos desconcierta y al mismo tiempo nos invita a repensar nuestras prácticas, a producir conocimientos desde otros formatos pero siempre desde una convicción compartida: el trabajo en equipo, en el que cada una de nosotras aporta desde una mirada, una trayectoria en la cátedra que se complementa a partir del diálogo y la confianza, el compartir dudas y esperanzas. Todo un ejercicio de creatividad al interior del equipo y con y para con los y las estudiantes.

Las categorías de tiempo y espacio implosionaron, tomando otras dimensiones en el campo educativo. La corporalidad también entró y entra en otras dimensiones, como docentes, las jornadas laborales se volvieron más extensas, más exigidas. La vida cotidiana se alteró completamente, los tiempos laborales se vuelven difusos.

Los cuerpos, fraccionados en ventanas de una pantalla, fueron y son el soporte posible para generar encuentros y construir sentidos.

En este trabajo queremos compartir los debates al interior del equipo docente y con nuestros/as estudiantes en torno a la posibilidad misma de la práctica en las escuelas secundarias y al recorrido que podemos hacer desde la pantalla hasta tanto se "normalicen" las clases en la Universidad.

Las fuentes utilizadas son las biografías escolares de los/as estudiantes y los registros de tres actividades virtuales vía jitsi en las que nos "encontramos" con ellos y ellas y con docentes del nivel secundario que se desempeñan en diferentes escuelas de la Ciudad de La Plata y Gran La Plata.

Esos encuentros virtuales promovieron preguntas y debates que posibilitaron pensar el contexto desde voces que enuncian diversas experiencias.

Producir documentación pedagógica de este tiempo tan particular es uno de los objetivos que nos guía, objetivo no ajeno a la reflexión sobre la práctica en una mirada diacrónica con las experiencias propias y las de los estudiantes.

El concepto que nos permite articular las categorías de tiempo, espacio y sujetos, es el de cultura escolar, definido por Dominique Julia (2001) que refiere tanto a las normas como a las prácticas coordinadas a finalidades que varían según las épocas en un cronosistema que habilita hablar de cultura(s) escolar(es) en plural.

Es por demás evidente que este trabajo se produce al calor de una situación inédita y en ese sentido en él se traducen nuestras reflexiones en voz alta.

\section{PALABRAS CLAVE}

tiempo,

formación docente, pandemia, vida cotidiana 


\section{KEY WORDS}

time,

teacher training,

pandemic,

daily life

\section{ABSTRACT}

The certainties, or at least some from where we started to think about the classroom space, are in "recalculating" mode. Uncertainty invades us, confuses us and at the same time invites us to rethink our practices, to produce knowledge from other formats but always from a shared conviction: teamwork, in which each of us contributes from a perspective, a trajectory in the chair that is complemented by dialogue and trust, sharing doubts and hopes. A whole exercise in creativity within the team and with and for the students.

The categories of time and space imploded, taking other dimensions in the educational field. Corporeality also entered and enters other dimensions, such as teachers, the working hours became more extensive, more demanded. Everyday life was completely altered, working times become fuzzy.

The bodies, divided into windows of a screen, were and are the possible support for generating encounters and constructing senses.

In this work we want to share the debates within the teaching team and with our students about the very possibility of practicing in secondary schools and the path that we can take from the screen until classes are "normalized" in the College.

The sources used are the school biographies of the students and the records of three virtual activities via jitsi in which we "meet" with them and with teachers at the secondary level who work in different schools in the City of La Plata. and Gran La Plata.

These virtual meetings promoted questions and debates that made it possible to think the context from voices that enunciate diverse experiences.

Producing pedagogical documentation of this very particular time is one of the objectives that guides us, an objective that is not alien to reflection on practice in a diachronic view with the own experiences and those of the students.

The concept that allows us to articulate the categories of time, space and subjects is that of school culture, defined by Dominique Julia (2001), which refers both to norms and to coordinated practices for purposes that vary according to the times in a chrono-system that enables talking about school culture (s) in plural.

It is also evident that this work is produced in the heat of an unprecedented situation and in that sense our reflections are translated aloud. 


\section{PRESENTACIÓN}

El ciclo lectivo de Planificación didáctica y prácticas de la enseñanza en Historia se inicia este año de un modo particular, no en el aula con los y las estudiantes sino en el campus virtual de la Facultad de Humanidades y Ciencias de la Educación y en las cuadrículas de las pantallas que nos ofrecen los sistemas zoom y jitsi. Esta situación nos desestabiliza y nos conduce a una y otra "reunión virtual" para dialogar caminos, alternativas posibles, todos atravesados por el deseo de volver al aula. Prontamente advertimos que esa vuelta está en un horizonte por ahora inalcanzable.

Comenzamos preguntándonos por nuestro presente, ese aquí y ahora en el que el trabajo docente se entremezcla con el cotidiano de organización del día a día en nuestros propios hogares y familias. En esas conversaciones aparecen los efectos de la cuarentena y luego la inevitable pregunta ¿cómo seguir con esta cátedra de Planificación didáctica y prácticas de la enseñanza en Historia?, teniendo en cuenta que es la práctica misma el corazón de la cursada. Suponemos que los y las estudiantes no son ajenos a esta preocupación.

Nuestras conversaciones se centran en la pregunta ¿cómo pensar la cultura escolar $^{1}$ en este nuevo escenario que Pablo Pineau (2020) define como de experimentación social y educativa? en el intento de habitar esta nueva realidad socioeducativa y construir nuevos espacios y vínculos pedagógicos.

J.L. Borges escribe "El pasado es arcilla que el presente labra a su antojo. Interminablemente". El tiempo es la materia prima de nuestra disciplina, la dimensión que atraviesa nuestra condición humana. La propia vida genera imágenes y representaciones mentales de los escenarios sociales que se caracterizan por su doble dimensión: temporal y espacial. A lo largo de la cursada hacemos foco en la conciencia histórica en tanto competencia aglutinante que permite articular tres tipos de acciones, en tres tiempos y en una variedad de espacios continuos y discontinuos: percibir, comprender y orientarse. Percibir y comprender lo estático y lo cambiante, la velocidad y la lógica de los cambios, la densidad de cada tiempo, y orientarse para tomar decisiones para

1 Dominique Julia (1995) conceptualiza el concepto de Cultura Escolar como "un conjunto de normas que definen los saberes a enseñar y los comportamientos a inculcar, y un conjunto de prácticas que permiten la transmisión y la asimilación de dichos saberes y la incorporación de estos comportamientos" (Julia, 2001:9). 
el futuro. Y es precisamente esta conceptualización la que se instala en la densidad de este presente de cuarentena. La(s) urgencia(s) y la inmediatez que impone este particular tiempo -¿de excepción?- para dar respuestas ante el pasaje que supone la educación presencial a otra a distancia, contribuyen a que se experimente muchas veces incierto y abrumador. Su contracara, la quietud, el enlentecimiento de la vida individual y social también hacen que sea percibido del mismo modo. El pendulante ritmo de los cambios pareciera ser la única constante de esta nueva temporalidad que estamos transitando.

El aula no es solo un lugar donde compartimos conocimiento, sino también experiencias, aquello que pasa y nos pasa en ese contar contarnos aparecemos las/los sujetos con toda nuestra historia personal y colectiva. Ese equipo de cátedra que constituimos está atravesado por el contexto en qué nuestra práctica/vida se desarrolla y desde aquí nos propusimos compartir con los y las estudiantes nuestras miradas en este tiempo poblado de pantallas, de tanta virtualidad que agobia.

¿Cuáles son las coordenadas del nuevo espacio que transitamos? Pareciera que la ruta conocida se nos escurre entre los dedos, pensamos el aula y ya no es tal cual la conocíamos. La pantalla se nos presenta plagada de rectángulos con iniciales o con nombres, un diagrama (¿plano?) que cambia minuto a minuto, no siempre los nombres están en el mismo lugar. El espacio virtual se puebla de sonidos no deseados, de delate, de micrófonos que no se escuchan. Qué lejos está la pantalla de la descripción que hace el Principito cuando habla con el geógrafo

\begin{abstract}
¿Cuáles son las coordenadas del nuevo espacio que transitamos? Pareciera que la ruta conocida se nos escurre entre los dedos, pensamos el aula y ya no es tal cual la conocíamos. La pantalla se nos presenta plagada de rectángulos con iniciales o con nombres, un diagrama (¿plano?) que cambia minuto a minuto, no siempre los nombres están en el mismo lugar.
\end{abstract}

Exactamente -dijo el geógrafo- pero no soy explorador. Carezco totalmente de exploradores. No es el geógrafo quien va a contar las ciudades, los ríos, las montañas, los mares, los océanos y los desiertos. El geógrafo es demasiado importante para andar paseando. No abandona su escritorio. Pero en él recibe a los exploradores. Los interroga y toma nota de sus recuerdos. Y si los recuerdos de alguno de ellos le parecen interesantes, el geógrafo hace hacer una encuesta sobre la integridad moral del explorador. (Saint Exupery 2003:18) 
Seguramente nosotras somos ahora las exploradoras de este nuevo "mundo áulico" que se nos presenta como una pantalla con muchos rectángulos e iniciales, en ocasiones los nombres. No solo para nosotras con una historia como docentes en aulas "tradicionales" este espacio es desconcertante sino también para los estudiantes.

Al mismo tiempo acudimos en esta experiencia a las voces de diversos actores educativos, sujetos históricos que con sus voces, testimonios, pequeños relatos dan cuenta de sus experiencias pasadas como estudiantes, docentes, talleristas en escuelas, bachilleratos populares, facultades, cárceles. Narrativas que expresan diversas subjetividades de este tiempo, haciéndose, haciéndonos nuevas preguntas.

La vida cotidiana de cada uno/a de nosotros/as, esa experiencia del diario vivir, en este tiempo y espacio tan particulares, implica velocidades y frecuencias, un borroso itinerario que se despliega dando sentido a nuestras vidas. Esa textura de lo cotidiano que luego nos permite establecer relaciones con el escenario político, social y económico. (O Donnell).

En ese contexto es que decidimos compartir, por las ventanas de una aplicación virtual (jitsi), pinceladas de nuestras vidas cotidianas, cotidiano diferente a aquel previo al 20 de marzo, y en las cuales los conceptos tiempo/s, el espacio/s y sujeto/s nos interpelan de modo diferente.

Las "escenas" se suceden, cada uno/a se presenta con su nombre, datos del lugar en que se encuentra. Entre las docentes la escena que predomina es la "vorágine" en el cotidiano por el ritmo que adquirieron los encuentros con los/as alumnos/as a través de las pantallas, las horas de ese teletrabajo al mismo tiempo que nos preguntamos por nuestra historia en las prácticas, los nuevos horizontes que aparecen y las continuas demandas de cuidado, limpieza, conexión a internet, uso de la computadora. Estas pinceladas son compartidas por todas y todos los que aportan al encuentro: la falta de ganas de estudiar, la duda, sentirse perdido, desconcertado, tener sentimientos cruzados, "odiar" los trabajos prácticos. Se hace fuerte en las intervenciones el sonido de una palabra que adquiere un tono grave, implacable la vivencia de la INCERTIDUMBRE, aquietada por alguna voz más optimista.

Las "escenas" se suceden, cada uno/a se presenta con su nombre, datos del lugar en que se encuentra. Entre las docentes la escena que predomina es la "vorágine" en el cotidiano por el ritmo que adquirieron los encuentros con los/as alumnos/as a través de las pantallas, las horas de ese teletrabajo al mismo tiempo que nos preguntamos por nuestra historia en las prácticas, los nuevos horizontes que aparecen y las continuas demandas de cuidado, limpieza, conexión a internet, uso de la computadora. 
A partir de todo lo dicho anteriormente en este trabajo desarrollamos el inicio del recorrido de la cátedra en tiempo de pandemia utilizando como fuentes las biografías escolares, el encuentro virtual con los y las estudiantes y docentes de historia que se desempeñan en diferentes instituciones de la ciudad de La Plata, fuentes de las que surgen las inquietudes que transitan en este tiempo, definido por una de las estudiantes como "puro presente".

\section{Un camino que se hace al andar}

La primera pregunta que año a año nos hacemos surge una vez más con una fuerza inusitada: ¿Quiénes son nuestros/as estudiantes? Conocemos sus nombres a través de la lista de inscripción, su presentación en el campus de la materia, por los nombres que aparecen en la pantalla o sus caras en las fotografías o videos, en caso que recurran a ellas o enciendan la videocámara.

Decidimos iniciar la cursada virtual con sus biografías escolares, las que en este presente adquieren otro sentido ya que la rememoración de la escuela, la facultad conocida se hace lejana de la experiencia del presente atravesada por la virtualidad, donde los vínculos no son cara a cara, gesto a gesto. Aprendemos esos vínculos desde las narraciones que docentes y estudiantes construimos en el campus con la escritura de las clases, las intervenciones en los foros y las "conversaciones virtuales".

Año a año la biografía escolar es uno de los primeros trabajos que encuadra e inicia la cursada de la materia: Planificación didáctica y prácticas de la enseñanza en Historia porque la consideramos un ejercicio reflexivo en la medida que la investigación sobre formación docente recupera este aspecto (Davini, 1995; Diker y Terigi, 2008). Desde este marco la escuela aparece rememorada en las biografías escolares de los practicantes de Historia con anécdotas y algunas fotos. A través de ellas podemos vislumbrar múltiples cuestiones: contenidos, recursos, prácticas hegemónicas, estilos docentes, decisiones historiográficas, modos de enseñar, aprender, la escuela y las relaciones de poder, género, etc.

Parafraseando a Inés Dussel (2020) "la clase en pantuflas" acarrea otros desafíos de los habituales o por lo menos de los más o menos conocidos. Se abren e inauguran otros desafíos por parte de nosotras como docentes, repensar y reactualizar un qué enseñar y cómo enseñarlo en tiempos de pandemia, tiempos aparentemente aquietados, estos tiempos líquidos (Bauman, 2007) plagados de incertidumbres conviven con tiempos inmediatos (mails, llamadas telefónicas, grupo de wpp laborales que aguardan respuesta) y una tensión cotidiana y permanente entre tiempos personales y profesionales, donde no se sabe cuándo empieza y termina el tiempo del docente, el tiempo de trabajo y el tiempo personal. Pareciera que el espacio único del hogar ha borrado todas las fronteras temporales y espaciales que tienen los diferentes roles que asumimos cotidianamente. 
Otra cuestión relevante en esas biografías son las descripciones referidas a la enseñanza de la Historia. Su análisis pormenorizado en las clases brinda pistas para pensar el propio estilo de enseñanza a la vez que impulsa la comparación de esas experiencias con las de los practicantes y docentes que la cátedra convoca para un espacio de debate virtual titulado experiencias en diálogo.

Se abren e inauguran otros desafíos por parte de nosotras como docentes, repensar y reactualizar un qué enseñar y cómo enseñarlo en tiempos de pandemia, tiempos aparentemente aquietados, estos tiempos líquidos (Bauman, 2007) plagados de incertidumbres conviven con tiempos inmediatos (mails, llamadas telefónicas, grupo de wpp laborales que aguardan respuesta) y una tensión cotidiana y permanente entre tiempos personales y profesionales, donde no se sabe cuándo empieza y termina el tiempo del docente, el tiempo de trabajo y el tiempo personal.

En las escenas relatadas en las biografías aparecen actividades recurrentes tales como cuestionarios, síntesis, líneas de tiempo al mismo tiempo que aparecen las relaciones pasado presente, los debates sobre diversos tópicos, la puesta en tensión de prejuicios y la explicación del profesor como central en la clase, pero asumiendo diversas modalidades, en algunos casos privilegiando vínculos afectuosos y en otros privilegiando los datos como saberes centrales a ser enseñados y aprendidos. El análisis de las fuentes que en este trabajo se describen corroboran los dichos de Paula González cuando expresa" la enseñanza de la historia está dando muestras de sentirse interpelada y responder -con mayor o menor fragilidad y fortaleza- a nuevos rumbos. Y es que la historia, como disciplina escolar, es una construcción histórica. Y como historiadores sabemos que en ese proceso habrá contradicciones, rutinas, rupturas, inercias, idas y vueltas" (González, 2013: 19).

¿Qué nuevos sentidos pueden los y las estudiantes dar a estas experiencias en este escenario de experimentación social y educativa?

Seguidamente, interpeladas por nuestro presente, nos preguntamos si esa escuela rememorada ¿Se parece a la escuela de hoy? ¿La escuela siempre fue igual? ¿Está en crisis? ¿Qué preservar y qué modificar de la escuela que conocimos? ¿Qué sucede en la escuela hoy? ¿Cómo atraviesa la pandemia a sus diferentes actores? ¿Cómo democratizar los vínculos al interior de la escuela? ¿Es posible una educación sin escuelas? ¿Es posible pensar los tiempos actuales como tiempos de oportunidad? 


\section{Experiencias en diálogo}

Este primer encuentro nos invita a promover dos encuentros virtuales más con practicantes de otras cohortes y docentes en ejercicio.

El 6 de mayo estuvieron presentes dos practicantes de la cohorte 2019 que se desempeñan como docentes en el FINES que funciona en la escuela de Abasto $^{2}$ y dos profesores de historia (que cursaron la materia en los años 2014 y 2017) quienes tienen a su cargo talleres en diferentes Unidades carcelarias ${ }^{3}$. El 8 de mayo contamos con la presencia en jitsi de cuatro profesores de Historia que trabajan en diferentes instituciones. Ivana en dos escuelas privadas, Sofía en un un CENS y en un Fines, Ignacio en una escuela pública no céntrica de Altos de San Lorenzo y Silvio en una escuela pública de Gonnet y en un colegio dependiente de la UNLP.

Muchos de los ex practicantes convocados se encuentran dando clases y comparten sus ideas respecto a cómo está afectando sus prácticas la pandemia. Aylén cuenta que el Bachillerato popular de adultos funciona como un plurigrado y de manera multidisciplinaria. Ahora dan clases a través de WhatsApp pero las familias tienen un celular por hogar y pocos datos. Comenta que no se enseñan contenidos "en una forma tradicional" sino se plantean temas por semana y se busca el modo de que se trabajen de manera interdisciplinaria. Que este año eligieron como eje Economía popular. Dice que la virtualidad en teoría está buena pero en la práctica es muy difícil. Julia amplía esta idea al mencionar que la pandemia refuerza más que nunca la desigualdad. Habla de las particularidades de les estudiantes de este bachillerato popular. "Son trabajadores hortícolas, migrantes, particularmente bolivianes y paraguayes, y no tienen acceso a insumos para prevenir el Covid19."

Dice que la virtualidad en teoría está buena pero en la práctica es muy difícil. Julia amplía esta idea al mencionar que la pandemia refuerza más que nunca la desigualdad. Habla de las particularidades de les estudiantes de este bachillerato popular

En su presentación, muy entrecortada y limitada por problemas técnicos manifiestan la realidad con la que se encuentran al enseñar en este contexto de pandemia. Ivana trabaja con estudiantes de clase media / media alta, que todos tienen conectividad. Nos comenta que las escuelas donde trabaja han optado por el trabajo en plataformas, una utiliza la Edmodo que es gratuita, "el colegio no paga" y otra utiliza Microsoft Teams, la brinda un padre que trabaja en esa empresa. Ivana cuenta que tiene que cumplir el horario presencial desde la virtualidad y llevar ade-

1 Ambas docentes forman parte de la Escuela Popular Tinku que funciona en la Escuela n 70 de Abasto. 2 Uno de ellos trabaja con un Taller de escritura en la Unidad Carcelaria 8 de Mujeres en la localidad de Los Hornos, partido de La PLata. 
lante clases por Zoom además de usar las plataformas. Ivana comenta que obviamente dar clases virtuales depende del contexto, implica muchas cosas. -"Pero ahora en privado también hay problemáticas, como la apatía, no responden a las clases por Zoom. En cambio los nenes de primero se quejan de que la clase de 40 minutos es corta"-. Agrega que las escuelas piden respetar los horarios pero que los chicos ahora tienen otros, son más noctámbulos y duermen hasta tarde.

El contexto en el que trabaja Ignacio solo es posible enviar los ejercicios y establece una diferencia entre ciclos señalando que $2 / 3$ de los estudiantes de 4to, 5to y 6 to entregan los trabajos, pero que los alumnos de 1 ro a 3 ro casi ni se conectan. Entre las dificultades encuentra la limitación de un celular por familia y no contar con internet por wifi sino solo por datos. "Me han dicho que deje de dar trabajos" y agrega que los chicos de la zona necesitan comida para lo cual la escuela realiza entregas periódicas de bolsones de alimentos provenientes del Estado al mismo tiempo que deben sortear numerosos problemas como por ejemplo recientes incendios en dos hogares. De este modo para este profesor no está resultando el plan de continuidad pedagógica. En tanto Sofía, encuentra similitudes entre la realidad que describe lgnacio y la que viven muchos de sus estudiantes. Pero señala con mucho énfasis "¡No estoy dando clase! Para mí esto no es dar clases". Si bien en los primeros 15 días de aislamiento "había como una euforia de productividad. Después el aislamiento comenzó a mezclarse con otras problemáticas". Entre esas problemáticas destaca las diferencias de clase, con respecto a la conectividad y la virtualidad y también aclara que en su caso la relación con les estudiantes está mediada por la preceptora. -"Intenté mandar vídeos y no muchos los pueden ver. Entonces empecé a trabajar desde sus posibilidades y no desde las mías. Y ni que hablar de las disposiciones psicológicas y emotivas. El contenido pasó a segundo plano. Entregué tres trabajos desde que comenzaron las clases. El contacto semanal es por audio para saber cómo están y es a través de la preceptora. ¡Esto no es dar clases! El primer trabajo fue que se presenten y yo me presenté. Hay un solo chico que se maneja conmigo por mail". Por su parte Silvio señala, entre cortes de internet que se producen en su dispositivo: “Lo que puedo transmitirles desde mi experiencia en esta situación fue encontrarme con un montón de emociones". Relata apenado que llegó a conocer a sus alumnos/as y en esa misma semana se produce la suspensión de las clases. Para describir esa situación dice: "La primera semana fue como echar una botella al mar". Hace hincapié en el trabajo que supone este nuevo formato y el desajuste entre una clase real en un aula y una clase virtual.

Vuelve a sumarse Ignacio y reitera que los sectores con los que trabaja no tienen acceso ni a la conectividad ni a la tecnología y que la escuela es el lugar donde se escucha a los estudiantes, donde ellos se sienten bien. Y se pregunta: - ¿¿Cuántas veces damos trabajos? ¿Y cuántas veces realmente pueden hacerlo en sus casas?"-. Indica que las actividades las realizan en el aula en la escuela. Da a entender dos 
problemáticas: el acceso a la tecnología y la falta de acompañamiento de los estudiantes, es un momento de soledad. Y le parece una utopía intentar que los estudiantes realicen sus tareas en sus casas por esta situación de cuarentena. En su voz se resalta justamente la ausencia del momento donde la clase y la escuela son un espacio de trabajo y de encuentro, que no se reemplaza con dar tareas.

Y en ese sentido aparece también el rol del docente y los desafíos del presente. Para Sofía la idea de la continuidad pedagógica como política educativa la contenta pero ve muchas falencias en los equipos directivos de las instituciones. -"No veo que la continuidad pedagógica sea de cualquier modo"-. Como positivo rescata el trabajo docente y que los docentes respondan a pesar de todo lo que a ellos también les está pasando, "con mucha voluntad y responsabilidad por ese Otro con el que formamos parte de algo. No somos héroes, los estudiantes tampoco y nadie te va a aplaudir a las 20 hs. Rescato la responsabilidad y el compromiso frente a este escenario".

Silvio toma la palabra para suscribir a lo dicho por Sofía y señala que la pandemia dejó a la luz las evidencias que teníamos los docentes con respecto a la tecnología en la educación. -"Su lugar no va a ser tan fundamental" afirma que "va a estar bueno cuando nos podamos juntar de nuevo en las escuelas para discutirlo" y enfatiza que: "Toda nuestra rutina está subvertida como la de todos los trabajadores. Es un momento para aprender con los estudiantes. Creo que se pone en evidencia el rol que cumplimos como el de otros trabajos y trabajadores. La escuela virtual es rara pero es una experiencia"-.

\section{CONCLUSIONES}

Muchas son las preguntas que se formulan los diarios, los especialistas y también nosotras y les estudiantes. En estos primeros pasos surge la certeza, tal como también sostienen muchos especialistas, de la necesidad de mantener el vínculo. Pero mantener el vínculo nos desafía a pensar el por qué, para qué y qué Historia enseñar en este contexto. Preguntas que no constituyen una ruptura con aquello que venimos pensando desde hace tiempo atrás y que las reformas educativas y diseños curriculares se formulan. Se trata de aquellas preguntas que, tal como sostiene Liliana Trigo (2007), funcionan desde hace décadas como los núcleos problemáticos de las Didácticas Específicas.

Es por eso que nos preguntamos ¿Qué rescatar de la "vieja escuela"?¿Por qué todo tiene que ser rupturas con el mundo que imaginamos a futuro? ¿Cuáles podrían ser las continuidades? ¿Enseñar Historia no sigue siendo la posibilidad de pensarnos "a caballo de los tiempos"? y al compás de una pregunta como latigillo ¿Cómo concretar una educación que posibilite de algún modo no profundizar las diferencias en una sociedad profundamente desigual? La escuela y los docentes no podemos solos, es imprescindible un colectivo que accione y luche en ese sentido. Viejas preguntas para pensar nuevos contextos.

[1] Cabe aclarar que esta reunión fue muy intervenida por problemas técnicos de todos los participantes y que hubo momentos se perdía el ida y vuelta. 
¿Cómo concretar una educación que posibilite de algún modo no profundizar las diferencias en una sociedad profundamente desigual? La escuela y los docentes no podemos solos, es imprescindible un colectivo que accione y luche en ese sentido. Viejas preguntas para pensar nuevos contextos. 


\section{BIBLIOGRAFIA}

BAUMAN, Z.(2006). “Modernidad líquida”. Buenos Aires: FCE.

DAVINI, M. C. (1995). "La formación docente en cuestión: política y pedagogía". Buenos Aires: Paidós.

DIKER, G. y TERIGI, F. (2008). “La formación de maestros y profesores: hoja de ruta". (1 ed. $3^{a}$ reimp). Buenos Aires: Paidós.

JULIA, Dominique (2001). "A cultura escolar como objetivo histórico". Revista Brasilera de História da Educação n 1, 9., 2001, 43.

PINEAU, P. ( 22/04/2020). ¿¿Y si la escuela para de golpe? Comentarios sobre la actual situación educativa". Compartido en el muro de Facebook de la Asociación Argentina de Historia de la Educación. Disponible en: https://www.facebook.com/ sahe95/posts/2937021459674526?_tn__=K-R

TRIGO, L. (2007). “El lugar de la Didáctica Específica de la geografía en la formación docente". En: Gema Fioriti y Patricia Moglia (comp.) La formación docente y la investigación en Didácticas Específicas. Cuadernos del CEDE: Universidad Nacional de San Martín.

SAINT-EXUPÈRY, Antoine (2003). "El Principito". Biblioteca Virtual de la UEB http://www.ueb.edu.ec 